\title{
Sonic hedgehog ligand partners with caveolin-1 for intracellular transport
}

\author{
Hua Mao ${ }^{1}$, Anna Mae Diehl ${ }^{1}$ and Yin-Xiong $\mathrm{Li}^{1,2,3, *}$
}

Prenatal alcohol exposure is the most common environmental factor leading to congenital birth defects in the United States. Although significant progress has been made in this field, the detailed molecular pathology of fetal alcohol syndrome (FAS) remains to be determined. Previously, we have shown that alcohol exposure perturbs hedgehog signal transduction in zebrafish embryos by inhibiting the post-translational cholesterol modification of Sonic hedgehog (Shh), leading to decreased levels of mature Shh ligand that is associated with the plasma membrane, and causing transient loss of Hh signaling, resulting in permanent FAS-related morphological abnormalities. In the present study, we further elucidate the mechanisms that regulate the intracellular transportation and secretion of Shh using the hepatic stellate cell line HSC8B. We have found that Shh is associated with caveolin-1 in the Golgi apparatus to form protein complexes and that these complexes are packaged as large punctuate structures (transport vesicles) that are transported to the plasma membrane in lipid raft microdomains. Alcohol exposure does not significantly interrupt translation of shh mRNA in endoplasmic reticulum (ER) or the trafficking of Shh from the ER to the Golgi apparatus. However, alcohol does prevent the entry of Shh into transport vesicles from the Golgi to the plasma membrane and specifically decreases the amount of caveolin-1/Shh complex found in lipid rafts, causing cytoplasmic accumulation of Shh and leading to a deficiency of Shh ligand secretion into the extracellular matrix.

Laboratory Investigation (2009) 89, 290-300; doi:10.1038/labinvest.2008.163; published online 12 January 2009

KEYWORDS: Sonic hedgehog; caveolin; protein interaction; protein transport; FASD; alcohol

Alcohol consumption during pregnancy is one of the leading causes of human fetal teratogenic exposure, and can cause a variety of irreversible phenotypic abnormalities, generally referred to as fetal alcohol spectrum disorders (FASD). The most extreme of these disorders, fetal alcohol syndrome (FAS), is characterized by craniofacial malformations, physical and mental retardation, cardiac septal defects, and minor joint abnormalities. ${ }^{1}$ FASD is a significant clinical challenge and is also an important social problem. Although progress has been made toward delineating the mechanisms that contribute to alcohol-induced birth defects, significant questions remain. For instance, how does alcohol preferentially induce a spectrum of reproducible defects in developing embryo and why are these defects limited to specific organs?

Sonic hedgehog (Shh), an embryonic morphogen that initiates an evolutionarily conserved signal-transduction pathway, plays a central role in regulating cell proliferation, differentiation, and morphological patterning of tissues that are vulnerable in FASD during embryogenesis. ${ }^{2,3}$ This signaling pathway also helps maintain adult stem cells and plays a role in the initiation of tumorigenesis. We recently determined that alcohol inhibits post-translational cholesterol modification of Shh and simultaneously disrupts hedgehogrelated cell differentiation, organogenesis and morphological patterning.

In vertebrates, extracellular lipid-modified Shh glycoproteins alleviate Patched (Ptc)-mediated inhibition of the signal transducer Smoothened by binding to Ptc receptors. ${ }^{4}$ Smoothened signaling results in the decoupling of the negative regulator Suppressor of Fused $(\mathrm{Su}(\mathrm{fu}))$ from Gli transcription factors. ${ }^{5}$ These Gli transcription factors can then be translocated to the nucleus, where they induce increased expression of several genes, including $p t c,{ }^{6} n k x 2.2{ }^{7}$ and $p a x 6 ;{ }^{8}$ Gli transcription factors also repress the expression of several genes, including SFRP-1, SFRP-2, Mip1- $\gamma$, and Amh. ${ }^{9}$

\footnotetext{
${ }^{1}$ Division of Gastroenterology, Department of Medicine, Duke University Medical Center, Durham, NC, USA; ${ }^{2}$ Department of Cell Biology, Duke University Medical Center, Durham, NC, USA and ${ }^{3}$ Department of Pediatrics, Duke University Medical Center, Durham, NC, USA

*Correspondence: Dr Y-X Li, MD, PhD, Department of Medicine, Duke University Medical Center, Snyderman-GSRB I, Suite 1073, 595 LaSalle Street, Box 3256, Durham, NC 27710, USA.

E-mail: yinxiong.li@duke.edu

Received 30 October 2008; accepted 18 November 2008
} 
Proteolytic cleavage and cholesterol modification of the $45-\mathrm{kDa}$ Shh precursor protein, which is mediated by the carboxyl-terminal domain of the precursor itself, result in a cholesterol-modified N-terminal peptide that serves as the mature Shh ligand. ${ }^{10}$ The tightly regulated post-translational cholesterol modification of Shh is required for efficient Shh signal transduction, which plays a critical role in normal embryonic development. ${ }^{11}$

Lipid modifications of Shh are critical to its transport, and play key roles in establishing Shh concentration gradients in developing embryos. In addition to being modified by cholesterol, the $20-\mathrm{kDa} \mathrm{N}$-terminal peptide is modified by a covalent linkage of a fatty acid. ${ }^{12,13}$ These modified Shh signaling peptides form large multimers, which are packaged into micelles for long-range transport. ${ }^{14}$ Recent work has demonstrated that the activity and function of Shh largely depends on the fatty acid and sterol modification of the ligand. ${ }^{15}$ As Shh plays roles in many facets of embryogenesis, the profound effect that sterol modification has on Shh activity may explain some of the teratogenic effects caused by perturbed cholesterol biosynthesis. ${ }^{16}$ For example, mutations in genes that encode enzymes involved in cholesterol biosynthesis, as is seen in patients having Smith-Lemli-Opitz syndrome, ${ }^{17,18}$ and inhibitors of cholesterol biosynthesis, whether consumed by range sheep that eat native plants containing natural inhibitors or applied to early embryos in a laboratory setting, produce a spectrum of phenotypes similar to those found in embryos harboring mutations in genes that encode components of the Hh signal-transduction pathway. ${ }^{19}$ Similar congenital defects also occur in fetuses carried by women who drink alcohol during pregnancy.

Caveolin is a cholesterol-binding protein that can be sequestered in caveolae-small $(50-100 \mathrm{~nm})$ invaginations in the plasma membranes of many vertebrate cell types that are characterized by the coalescence of lipid rafts. Caveolin appears to play important roles both in signal transduction and in cholesterol transport. Intriguingly, these two functions may be interrelated. Caveolin, which is an important regulator of cholesterol trafficking, is required for the transport of newly synthesized cholesterol from the endoplasmic reticulum (ER) to the plasma membrane; therefore, increased caveolin expression causes an increased efflux of cholesterol to the plasma membrane. ${ }^{20,21}$ Roy et al. ${ }^{22}$ have identified a dominant-negative mutant form of caveolin that causes cholesterol to accumulate in cytoplasmic vesicles, resulting in decreased cholesterol content in plasma membranes. Caveolin can also modulate the activity of signal-transduction pathways. Excessive levels of caveolin-1 inhibit the mitogenactivated protein kinase signaling pathway, and reduced levels of caveolin-1 activity has the opposite effect. ${ }^{23,24}$ It has also been shown that caveolin can affect signal transduction by directly binding signaling receptors such as Ptc. ${ }^{25}$

We have recently shown that exposure of zebrafish embryos to low levels of alcohol during gastrulation blocks covalent cholesterol modification of Shh, leading to impaired hedgehog signal transduction and resulting in a dose-dependent spectrum of permanent developmental defects that closely resemble the spectrum of defects observed in patients having FASD. ${ }^{26}$ One key observation is that alcohol exposure leads to decreased Shh in the plasma membrane. In this study, we have focused on determining the biochemical and cellular mechanisms that lead to lower levels of mature Shh ligand in the plasma membrane after alcohol exposure. Tissue growth and differentiation are rapid during embryogenesis and the expression of Shh is temporospatially regulated; therefore, it is relatively difficult to study Shh intracellular trafficking in embryos. However, the rat hepatic stellate cell line, HSC8B, ${ }^{27}$ expresses high levels of Shh ligands that can be easily detected by western blot analysis, immunostaining, or ELISA assay. These features make this cell line an ideal in vitro model for studying the intracellular transportation of Shh. Using this cell line, we have determined that Shh interacts with caveolin-1 to form a protein complex that associates with lipid rafts. Alcohol exposure disrupts the intracellular transport of Shh from the Golgi apparatus to plasma membranes and leads to decreased Shh/ caveolin-1 located in lipid raft domains. These findings help elucidate the mechanisms responsible for the intracellular transport of Shh and provide further insights into the molecular mechanisms underlying alcohol-induced congenital disorders.

\section{MATERIALS AND METHODS}

\section{Preparation of Lipid Raft and Western Blot Analysis}

Cell membranes and lipid rafts were isolated as previously described with slight modifications. ${ }^{13,25,28}$ Briefly, rat hepatic cell pellets were washed three times with ice-cold PBS. Cells from a $60-\mathrm{mm}$ dish were lysed and collected in $1.5 \mathrm{ml}$ of lysis solution $\left(10 \mathrm{mM} \mathrm{NaHPO}_{4}\right.$ at pH $6.5,150 \mathrm{mM} \mathrm{NaCl}, 0.5 \mathrm{mM}$ PMSF, $1 \%$ Triton X-100, $2 \mu \mathrm{g} / \mathrm{ml}$ pepstatin A, $10 \mu \mathrm{g} / \mathrm{ml} \mathrm{leu-}$ peptin, and $5 \mu \mathrm{g} / \mathrm{ml}$ aprotinin) at $4{ }^{\circ} \mathrm{C}$, and homogenized in a Dounce homogenizer, followed by centrifugation at $1000 \mathrm{~g}$ for $10 \mathrm{~min}$. The supernatant was collected and adjusted to $1.4 \mathrm{M}$ sucrose, and then gently overlaid on top of equal volumes of $1.0 \mathrm{M}$ sucrose, $0.5 \mathrm{M}$ sucrose, or $0.1 \mathrm{M}$ sucrose in TNE buffer and then centrifuged at 25000 r.p.m. at $4{ }^{\circ} \mathrm{C}$ for $18 \mathrm{~h}$ in an SW55Ti rotor (Beckman Coulter). Sucrose gradient fractions $(500 \mu \mathrm{l})$ were collected and each fraction was added to an equal volume of pre-chilled 2\% Triton X-114; this solution was allowed to stand on ice for $30 \mathrm{~min}$. We loaded each fraction into a polyacrylamide gel to resolve the proteins by electrophoresis; the resolved proteins were transferred from the gel to nitrocellulose membranes for western blot detection of Shh by anti-Shh (sc-1194; Santa Cruz, CA) and anti-caveolin-1 antibodies (sc-894; Santa Cruz). Calnexin ER membrane markers (ab13504) and 58K Golgi markers (ab27043) were purchased from Abcam Inc. (Cambridge, MA). Secondary anti-goat antibody was purchased from Santa Cruz and anti-mouse and anti-rabbit antibodies were purchased from Jackson ImmunoResearch 
(West Grove, PA). We detected signals using an $\mathrm{ECL}^{\mathrm{TM}}$ kit (Amersham) and West Pico kit (Pierce Biotech, Rockford, IL).

\section{Immunoprecipitation Assays}

Immunoprecipitations were performed using the One-Step Complete IP-Western kit (GenScript). Equal amounts of each hepatic cell lysate were loaded onto a SDS-PAGE gel to allow determination of protein expression levels by western blot analysis. The lysates were precleared using protein $\mathrm{A} / \mathrm{G}$ PLUS-Agarose beads (Santa Cruz). Beads were removed by centrifugation at $3000 \mathrm{~g}$ for $5 \mathrm{~min}$. The precleared lysates were incubated with the appropriate antibody, either anti-Shh antibody (SC-1194; Santa Cruz), or anti-caveolin-1 antibody (sc-894; Santa Cruz), for $1 \mathrm{~h}$ before adding the protein A/G beads, followed by overnight incubation with rotation at $4{ }^{\circ} \mathrm{C}$. The next day, beads were washed three times with PBS, and the protein was eluted with $2 \times$ loading buffer. The immunoprecipitates were then subjected to $4-20 \%$ gradient SDS-PAGE (Bio-Rad, Hercules, CA) and subsequently transferred to nitrocellulose membranes. After the transfer, the membranes were pretreated and rinsed with pre-blocking buffer provided in the kit. Meanwhile, the solution was prepared and incubated with appropriate primary antibodies at 1:1000 dilutions (Antibodies for Shh and caveolin-1 are described above, anti-Ptc G-19 sc-6149; Santa Cruz); protein A\&G blocker was added to the solution to prevent leaked protein from the $\mathrm{A} / \mathrm{G}$ beads. The membrane was then washed three times with Tris-HCl-buffered saline $(10 \mathrm{mM}$ Tris- $\mathrm{HCl}$, $\mathrm{pH} 7.2,150 \mathrm{mM} \mathrm{NaCl}$, and $0.1 \%$ Tween 20 ), and then incubated with secondary antibody for $1 \mathrm{~h}$ at room temperature. Secondary antibody anti-goat (sc-2033) was purchased from Santa Cruz. Anti-mouse (NA931V) and anti-rabbit (NA934V) were purchased from Jackson ImmunoResearch. The signal was detected using the West Pico kit (Pierce Biotech) or the detection reagents provided in the One-Step Complete IP-Western kit.

\section{Immunohistochemistry}

Briefly, HSC8B cells cultured in the eight-well Lab-Tek ${ }^{\mathrm{TM}}$ Chambered Coverglass system (Nunc, Rochester, NY) were washed three times with PBS and then fixed with $4 \%$ formaldehyde for $20 \mathrm{~min}$, followed by incubation in $0.1 \%$ Triton X-100 for 5 min; cells were blocked in 10\% fetal bovine serum and $10 \%$ donkey serum for $1 \mathrm{~h}$, then incubated with antiShh (sc-1194; Santa Cruz) and anti-caveolin-1 (sc-53564, FITC; Santa Cruz), or anti-LAMP1 rabbit polyclonal antibody (lysosome marker, ab24170; Abcam Inc.) at 1:200 dilutions for $2 \mathrm{~h}$ at room temperature. Cells were washed three times with PBS, and incubated with either Alexa Fluor594conjugated anti-goat antibody (A11079; Invitrogen) or Alexa Fluor488-conjugated anti-mouse antibody (A21200; Invitrogen) for $45 \mathrm{~min}$. For ER and Golgi staining, the cells were fixed in methanol-free paraformaldehyde. The ER staining was performed using the SelecFX Alexa Fluor488
Endoplasmic Reticulum Labeling Assay kit (S34200; Invitrogen, CA). Golgi organelles were stained with an appropriate antibody (ab27043; Abcam Inc.) at a 1:200 dilution. After incubation with the secondary antibodies, the cells were washed five times with PBS, and Z-stack images were collected by Zeiss LSM 5LIVE confocal microscopy.

\section{Concentrating Proteins from Culture Medium and ELISA Assay}

HSC8B cells were cultured in DMEM medium with 10\% FBS. When cells reached $75 \%$ confluence, the culture medium was replaced with fresh DMEM containing 1\% FBS and various concentrations of alcohol and the cells were incubated for an additional $3 \mathrm{~h}$. After alcohol treatment, the cells were incubated in alcohol-free DMEM medium containing 1\% FBS for an additional $16 \mathrm{~h}$ to allow Shh protein to be secreted into medium. The collected media were first filtered through Millipore Centricon Plus-100 to remove proteins having molecular weights higher than $100 \mathrm{kDa}$, then concentrated using Centricon Plus-10, enriching for proteins with molecular weights ranging from 10 to $100 \mathrm{kDa}$. The enriched protein was used for western blot analysis (sc-1194; Santa Cruz) and ELISA assay analysis (Mouse Sonic Hedgehog ELISA development kit, DY461; R\&D System Inc.).

\section{Cell Apoptotic Activity}

Cell apoptosis was assayed using the Apo-ONE Homogeneous Caspase 3/7 Apoptosis Assay kit (G7791; Promega, Madison, WI) according to the manufacturer's instructions. ${ }^{29}$

\section{RESULTS}

\section{Caveolin-1 Binds with Shh to form a Protein Complex}

We have determined that alcohol exposure results in defective Shh cholesterol modification and impairs association of Shh with the plasma membrane, suggesting that alcohol causes a defect in the intracellular transport of this ligand. ${ }^{26}$ As a first step toward determining the mechanisms underlying Shh intracellular trafficking, we extracted proteins from HSC8B cells with non-ionic detergent and used density gradient ultracentrifugation to fractionate the protein extract and isolate proteins from different cellular compartments. ${ }^{13}$ After ultracentrifugation, we collected 17 individual gradient fractions (each fraction contained $500 \mu \mathrm{l}$ ), from the lowest density (at the top of the centrifuge tube) to the highest (at the bottom of the tube). The distribution of lipid raft proteins was primarily confined to fractions $4-11$, as indicated by the presence of the lipid raft-associated protein caveolin-1 (Figure 1a, bottom panel). In addition, we found that the distribution of Shh was confined to fractions $6-17$; Shh colocalized with caveolin-1 in fractions 6-11 (Figure 1a, top panel).

Physical colocalization of Shh and caveolin-1 in the gradient fractions suggests that these two proteins have some similar physical characteristics or that they functionally interact. As caveolin-1 plays an important role in protein and 


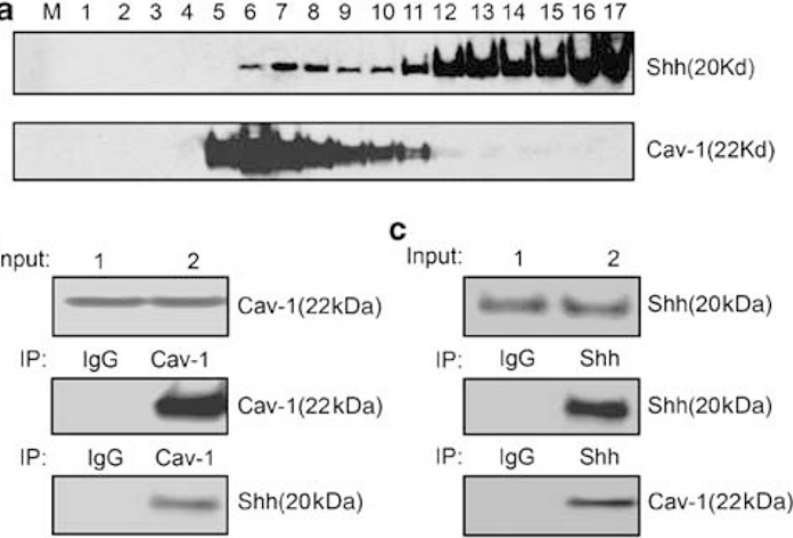

Figure 1 (a) Shh protein co-distributes with caveolin-1 in lipid raft fractions. Hepatic stellate cells were lysed in $1 \%$ Triton X-100 and subjected to raft isolation by sucrose density gradient ultracentrifugation. Fractions were analyzed by western blotting for Shh (top panel) and caveolin-1 (bottom panel). (b, c) Shh and caveolin-1 reciprocally co-precipitate suggesting close physical interaction. Total cell lysates and corresponding immunoprecipitates from beads loaded with control IgG and caveolin-1 antibodies (b) or control IgG and Shh antibodies (c) were analyzed by western blot for caveolin-1 and Shh as indicated.

cholesterol transport, and cholesterol modification is crucial for Shh trafficking, one intriguing possibility is that Cav-1 and Shh form a protein complex that is involved in intracellular trafficking of Shh. To test this possibility, we performed a series of immunoprecipitation assays on wholecell lysates isolated from the cell line HSC8B. We used an anti-caveolin-1 antibody to pull down proteins from the lysates, and then performed western blot analyses using anticaveolin-1 and anti-Shh antibodies. We confirmed that loading was approximately equal in each lane using the anticaveolin-1 antibody (Figure 1b, top panel). As expected, the anti-caveolin-1 antibody precipitated the $22-\mathrm{kDa}$ caveolin- 1 protein (Figure 1b, middle panel, lane 2). Furthermore, it precipitated the mature $20-\mathrm{kDa}$ Shh ligand (Figure $1 \mathrm{~b}$, bottom panel, lane 2), indicating that caveolin-1 physically associates with Shh in a protein complex (possible not directly). The negative control IgG antibody was unable to precipitate either caveolin-1 or Shh (Figure 1b, lane 1, middle and bottom panels). Moreover, we confirmed the interaction between Cav-1 and Shh using an anti-Shh antibody for immunoprecipitation assay to pull down its counterpart, Cav-1. The conditions used in this experiment paralleled those used in the previous immunoprecipitation assay; in this case, equal protein loading was confirmed using the anti-Shh antibody (Figure 1c, top panel). We found that the anti-Shh antibody immunoprecipitates both Shh (Figure 1c, middle panel) and caveolin-1 (Figure 1c, bottom panel) from HSC8B cell lysates. As anti-Shh antibodies and anti-caveolin-1 antibodies independently co-precipitate caveolin-1 and Shh from HSC8B cell lysates, it is likely that both caveolin-1 and Shh exist in a same protein complex in this cell line. a

Input (lipid raft)

$\begin{array}{llllllll}\text { A0 } & \text { A0 } & \text { A0.2 } & \text { A0.4 } & \text { A0.6 } & \text { A0.8 }\end{array}$

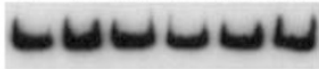

C IP:

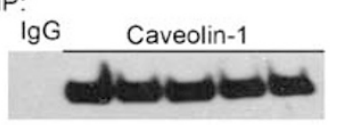

e IP:

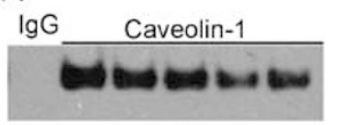

g IP:

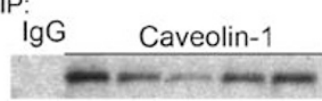

b Input (whole-cell lysate)

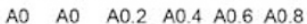

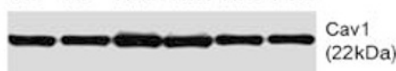

d

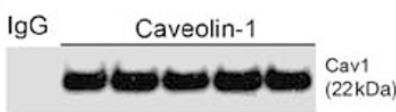

f

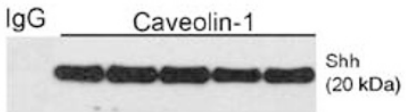

h

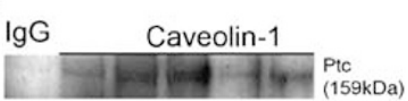

Figure 2 Alcohol exposure decreases caveolin-1/Shh complex levels in lipid raft fractions, but not in whole-cell lysates. Triton lipid rafts and total extracts were prepared from $\mathrm{HSC} 8 \mathrm{~B}$ cells treated with various alcohol concentrations (w/v: $0,0.2,0.4,0.6$, and $0.8 \%$ ) for $2 \mathrm{~h}$. Combined raft fractions (left) and total extracts (right) from cells at each alcohol concentration were directly probed for caveolin-1 (a, b) or subjected to immunoprecipitation with anti-caveolin antibody beads prior to western blot analysis for caveolin-1 (c, d), Shh (e, f), and Ptc (g, h). All experiments were repeated three times.

\section{Alcohol Specifically Decreases the Formation of the Caveolin-1/Shh Complex in Lipid Rafts}

The observation that Shh and caveolin-1 exist in a same protein complex raises an interesting question regarding our previous work. We showed that alcohol exposure does not affect total Shh levels, but rather results in defective Hh signal transduction by causing a dose-dependent decrease in the concentration of cholesterol-modified, mature Shh ligand at the cell plasma membrane. ${ }^{26}$ To determine whether this decrease in the plasma membrane-associated Shh ligand is specifically linked to the interaction between caveolin-1 and Shh, we investigated the effect of alcohol exposure on the interaction of caveolin-1/Shh in lipid rafts and in whole-cell lysates. As described in Figure 1, cellular proteins isolated by non-ionic, detergent-resistant extraction were fractionated by density gradient ultracentrifugation. ${ }^{13}$ Lipid raft-associated proteins were present in fractions 4-11, as indicated by the presence of caveolin-1 (Figure 1a, bottom panel and Figure 4a, second panel); we specifically performed co-immunoprecipitation assays on fractions containing lipid raftassociated proteins (fractions 7-9) using an anti-caveolin-1 antibody. HSC8B cells were exposed to various concentrations of alcohol $(0,0.2,0.4,0.6$, and $0.8 \% \mathrm{w} / \mathrm{v}$ corresponding to $0,43,87,130$, and $174 \mathrm{mM}$ ) for $2 \mathrm{~h}$ prior to protein extraction and density gradient ultracentrifugation. Fractions 7-9 from the density gradient were pooled and used in the immunoprecipitation assays. Prior to performing the assays, equivalent protein loading by western blot analysis was confirmed using an anti-caveolin-1 antibody (Figure 2a and $\mathrm{b}$ ). Anti-caveolin-1 antibody was used to 
immunoprecipitate proteins from the lipid raft (Figure 2a, c, e and g) or whole-cell lysate preparations (Figure $2 b, d, f$ and $\mathrm{h}$ ); and the immunoprecipitates were probed with the anticaveolin-1 (Figure 2c and d), anti-Shh (Figure 2e and f) and anti-Ptc antibodies (Figure $2 \mathrm{~g}$ and $\mathrm{h}$ ). The amount of caveolin-1 protein in the lipid raft and whole-cell lysate preparations was not significantly affected by alcohol exposure (Figure $2 \mathrm{c}$ and $\mathrm{d}$ ); however, alcohol exposure decreased the amount of caveolin-1-associated Shh in lipid raft fractions (Figure 2e), but not in whole-cell lysate preparations, in a dose-dependent manner (Figure 2f). Meanwhile, the amount of Ptc that was pulled down by anti-caveolin-1 antibody was not affected by alcohol exposure, either in the lipid raft fraction (Figure $2 \mathrm{~g}$ ) or in the whole-cell lysate (Figure $2 \mathrm{~h}$ ), suggesting that alcohol treatments do not increase the endocytosis of the Ptc/Shh/Cav-1 complex.

We also used immunohistochemistry to evaluate the effect of alcohol on caveolin-1/Shh complex formation. Double staining HSC8B cells with the anti-caveolin-1 antibodies (Figure 3a, green) and anti-Shh antibodies (Figure 3b, red) revealed a punctate, salt-and-pepper, colocalized distribution of Shh and caveolin-1 in the cytoplasm, particularly approximate to the plasma membranes (Figure $3 \mathrm{c}$, yellow as indicated by arrows). Exposure of the cells to $0.4 \%(\mathrm{w} / \mathrm{v})$
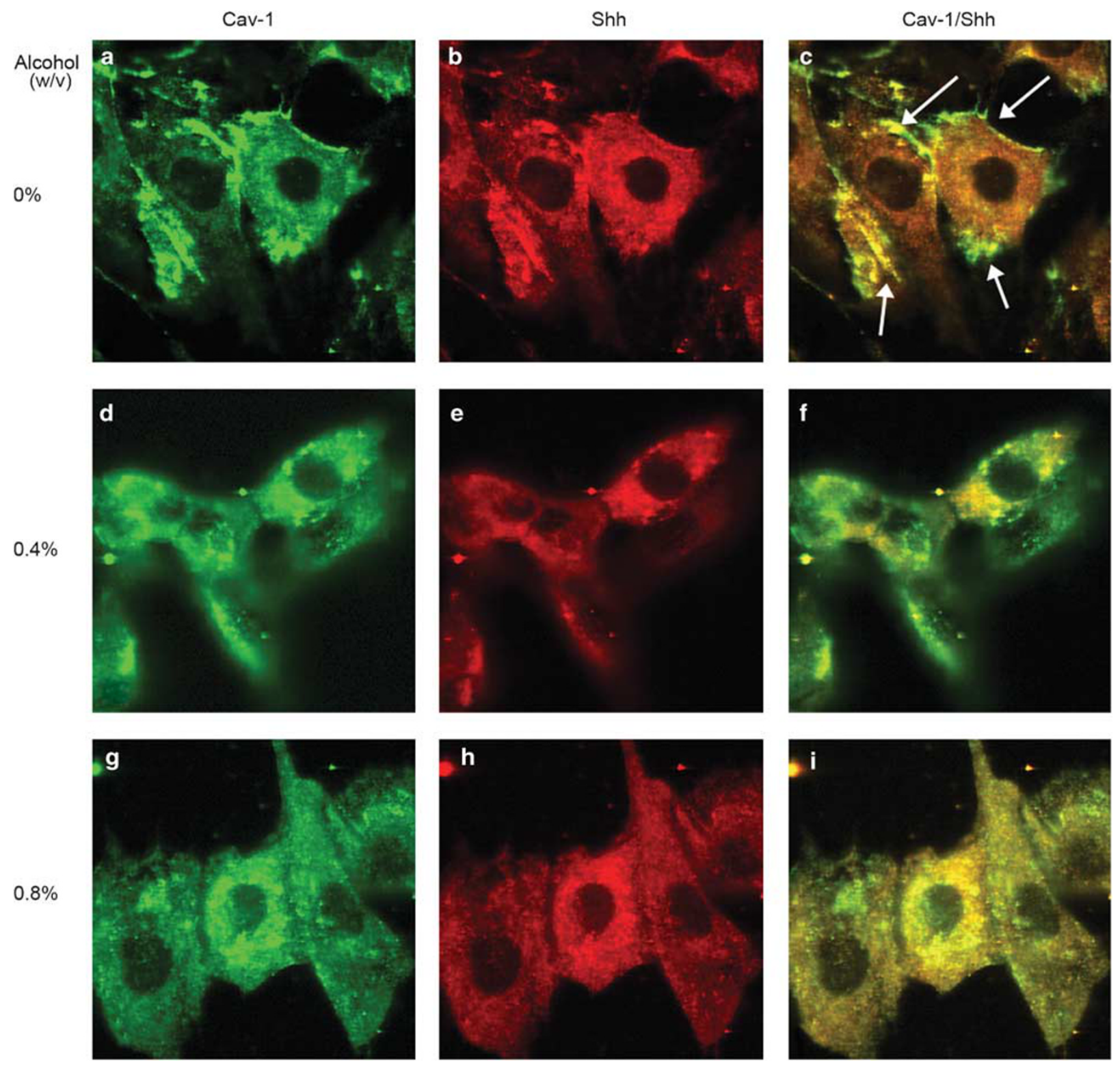

Figure 3 Immunohistochemistry revealed colocalization of caveolin-1 and Shh. In untreated hepatic stellate cells, caveolin-1 (a, green) and Shh (b, red) colocalized in the cytoplasm, and particularly in the cell plasma membrane (c, yellow, indicated by arrows). When cells were exposed to $0.4 \%$ (d-f) or $0.8 \%$ $(\mathbf{g}-\mathbf{i})(\mathrm{w} / \mathrm{v})$ alcohol for $30 \mathrm{~min}$, Shh levels were not affected; however, the punctate structures and amount of Shh colocalizing with caveolin-1 at the plasma membrane dramatically decreased. 
a

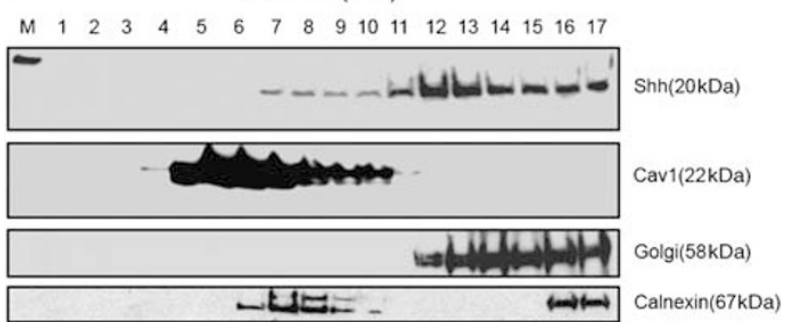

b

$\begin{array}{llllllllllllllllll}M & 1 & 2 & 3 & 4 & 5 & 6 & 7 & 8 & 9 & 10 & 11 & 12 & 13 & 14 & 15 & 16 & 17\end{array}$

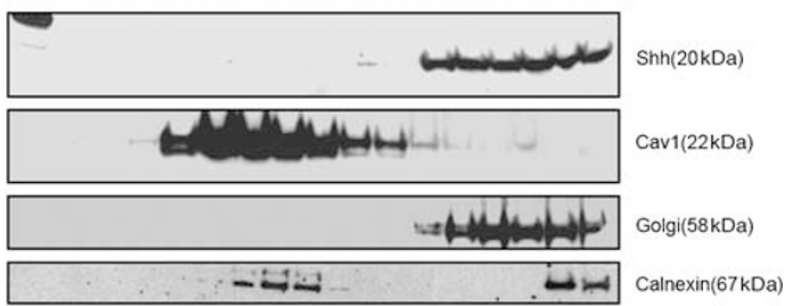

Figure 4 Alcohol disturbs Shh colocalization with caveolin-1 in lipid rafts and causes Shh to colocalize with markers for the Golgi and ER organelles. $\mathrm{HSC} 8 \mathrm{~B}$ cells were treated with or without $0.6 \%$ alcohol for $2 \mathrm{~h}$ prior to the lipid raft preparation. Raft fractions from control (a) or ethanol-treated (b) cells were analyzed for Shh, caveolin-1, a 58-kDa Golgi protein or the ER marker, calnexin, as indicated. Alcohol treatment causes loss of Shh from lipid raft fractions 7-11 and movement to denser fractions containing Golgi and ER markers.

(Figure $3 \mathrm{~d}-\mathrm{f}$ ) or $0.8 \%(\mathrm{w} / \mathrm{v})$ (Figure $3 \mathrm{~g}-\mathrm{i}$ ) alcohol for $30 \mathrm{~min}$ did not cause a noticeable change in either the total amount of caveolin-1 (Figure 3d and g, green) or of Shh (Figure 3e and $\mathrm{h}$, red). In contrast, the amount of punctate colocalized particles dramatically decreased in an alcohol dose-related manner, nearly disappearing from the cell plasma membranes at high alcohol concentrations (Figure $3 \mathrm{f}$ and i, yellow). Both biochemical and immunohistochemical analyses indicated that caveolin-1 and Shh form a protein complex, and that this complex enriches in lipid raft domains at the plasma membrane. Alcohol exposure disrupts the trafficking of the caveolin-1/Shh complex to plasma membranes, particularly into the lipid raft structures. These observations suggest that alcohol exposure causes a defect in the transport of the Shh ligand, resulting in decreased levels of secreted ligand.

\section{Alcohol Exposure Causes Defective Transport of Shh from the Golgi Compartment into Transport Vesicles and Leads to Shh Accumulation in the Cytoplasm}

To begin delineating the detailed molecular mechanisms that underlie the deleterious effect of alcohol on Shh transport in ligand-producing cells, we first compared the distribution of Shh among fractions generated by density gradient ultracentrifugation to the distribution of lipid rafts (caveolin-1), Golgi and ER compartment markers in the same fractions. In protein extracts isolated from cells not exposed to alcohol, western blot analyses indicated that Shh was broadly dis- tributed in fractions 7-17 (Figure 4a, top panel); in these extracts, lipid raft-associated proteins were distributed in fractions $4-11$, as indicated by the presence of caveolin-1 (Figure 4a, second panel). Golgi-associated proteins were present in fractions 12-17 (Figure 4a, third panel); and ERassociated proteins were located in fractions of 6-9, and 16 and 17 (Figure $4 \mathrm{a}$, bottom panel), as demonstrated by the presence of specific Golgi and ER markers. In protein extracts isolated from $\mathrm{HSC} 8 \mathrm{~B}$ cells that were exposed to $0.6 \%$ alcohol $(\mathrm{w} / \mathrm{v}, 130 \mathrm{mM}$ ) for $2 \mathrm{~h}$, the density gradient distribution of Shh was restricted to fractions 12-17 (Figure $4 \mathrm{~b}$, top panel). These fractions contain proteins associated with the Golgi and rough ER (Figure 4b, third panel), indicating that alcohol exposure shifts Shh distribution away from the compartments that contain lipid rafts (Figure $4 \mathrm{~b}$, second panel) and smooth ER compartments (Figure 4b, bottom panel).

To further elucidate the defects that alcohol exposure causes, we used confocal microscopy to examine cells stained with anti-Shh antibodies and antibodies generated against either Golgi or ER compartment markers. In untreated cells (A0; Figure 5, top panel), the ER marker (identified using an anti-PID antibody) (Figure 5a, left column, green) and Shh (Figure 5a, middle column, red) colocalized in the cytoplasm in large punctate structures (Figure 5a, right column, yellow); however, when HSC8B cells were exposed to 0.4 and $0.8 \%$ $(\mathrm{w} / \mathrm{v})$ alcohol for 1 or $2 \mathrm{~h}$ (Figure 5 , second to fifth panels), although the ER marker and Shh levels and their colocalization were unaffected, rather than many large punctate structures (transport vesicles), ${ }^{30}$ we observed a diffuse, homogeneous distribution pattern for Shh. Using the same experimental conditions, we determined whether the Golgi marker (Figure 5b, left column, green) and Shh (Figure 5b, middle column, red) colocalized by overlaying the corresponding images (Figure 5b, right column, yellow) and found that alcohol treatment did not significantly alter Shh distribution in the Golgi apparatus. Hence, the observation that Shh is distributed in a diffuse and homogenous manner indicates that the primary defect in Shh transport caused by alcohol exposure occurs during trafficking from the Golgi to the cell plasma membrane. To rule out that the accumulation of Shh in the cytoplasm is not due to increased endocytosis of the Ptc/Shh complex, we performed immunohistochemistry analysis using an anti-Shh antibody and a lysosome marker. Alcohol exposure slightly increased the amount of lysosome marker detected, but cytoplasmic Shh was distributed in a homogenous pattern in which most Shh protein was not located in lysosomal structures (data not show).

Previously, we demonstrated that alcohol exposure inhibits the post-translational modification of Shh by cholesterol, decreases the amount of the mature Shh ligand associated with the cell membrane, and leads to a spectrum of defects in our zebrafish model that are similar to the defects observed in patients having fetal alcohol syndrome. ${ }^{26}$ In this study, we have determined that alcohol exposure leads to defective intracellular transport of the Shh ligand. This is accomplished 
a
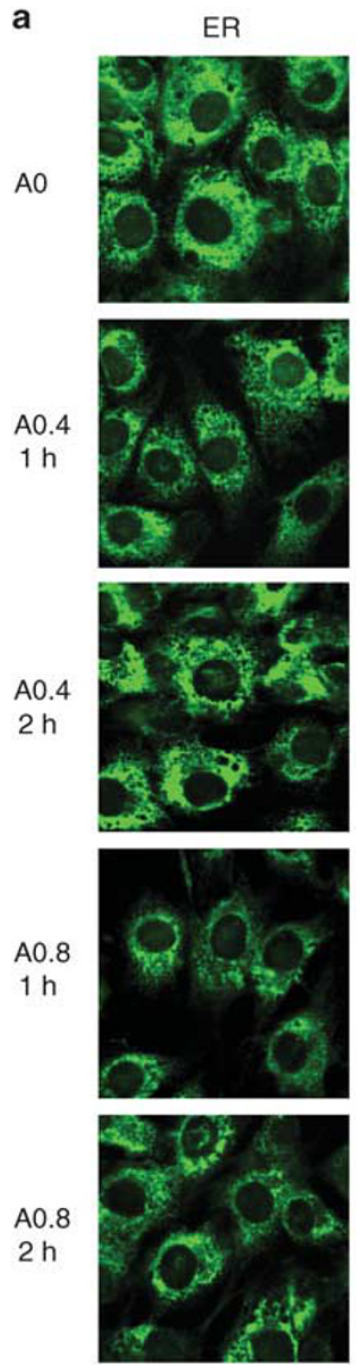

Shh
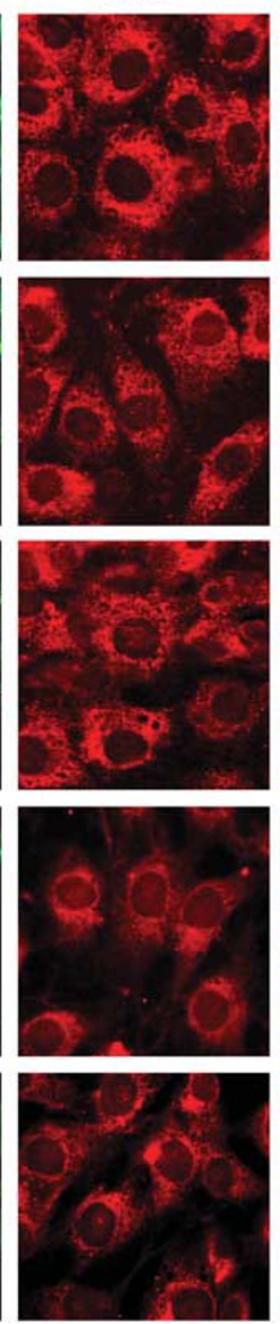

Merge
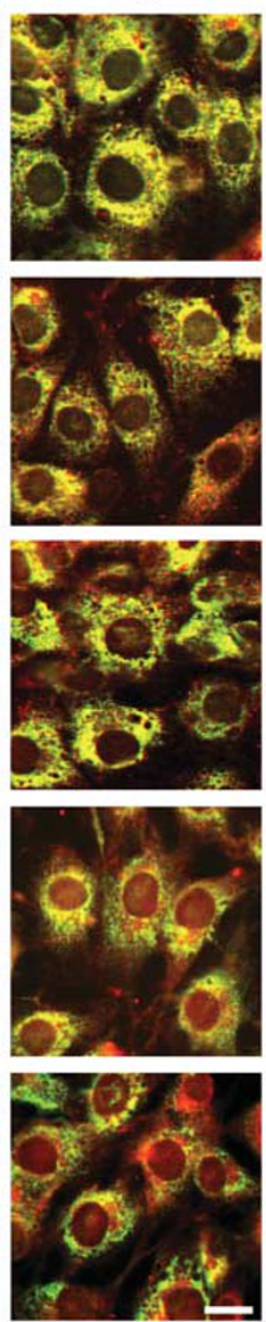

b Golgi
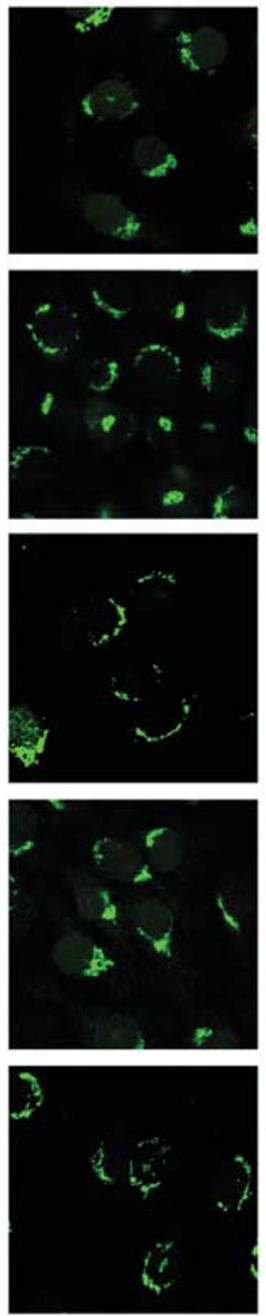

Shh
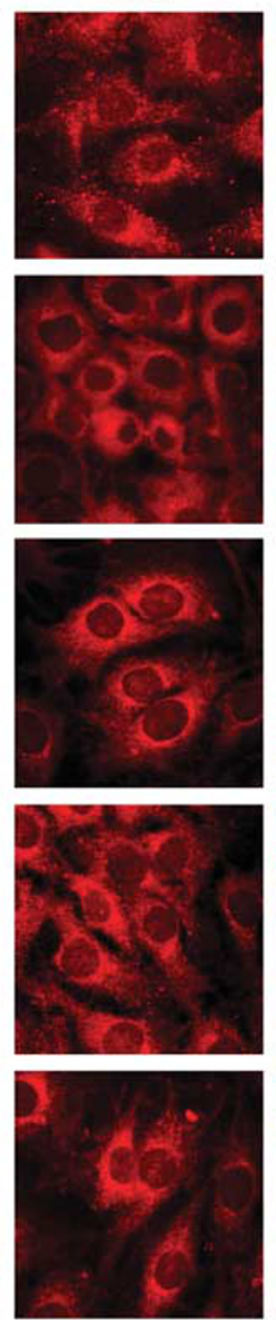

Merge
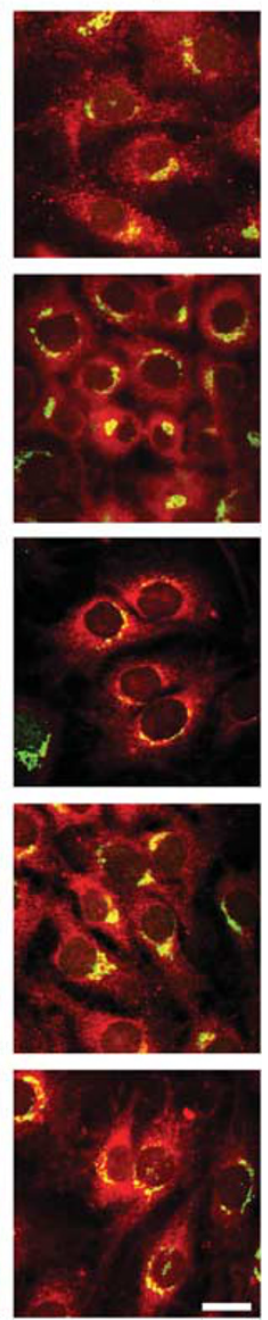

Figure 5 Alcohol exposure causes defective transport of Shh from the Golgi apparatus to the plasma membrane and leads to Shh accumulation in the cytoplasm. Cells were prepared for immunohistochemical analysis by co-staining with (a) ER marker (green) and Shh (red); or (b) Golgi apparatus marker (green) and Shh (red). In untreated cells (A0, top panel), some of the Shh proteins were located in ER and Golgi apparatus indicated by their colocalizations (yellow) of Shh and ER/Golgi markers; however, a lot of Shh proteins forms as a large punctate structures outside of the Golgi apparatus in the cytoplasm. When HSC8B cells were exposed to 0.4 and $0.8 \%(\mathrm{w} / \mathrm{v})$ alcohol for 1 or $2 \mathrm{~h}$ (second to fifth panels), although the amount of Shh (a, $\mathbf{b}$, middle column, red) and its localization in ER and its entry into the Golgi apparatus were unaffected (a, b, right column, yellow), rather than many large punctate structures (transport vesicles), it revealed a diffuse, homogeneous distribution pattern for Shh that is accumulated in the cytoplasm. Scale bar $=50 \mathrm{~nm}$.

by inhibiting the ability of the caveolin-1/Shh complexes entering into transport vesicles (large punctate structures), thus preventing transport from the trans-end of Golgi into the plasma membrane in lipid raft domains.

\section{Alcohol Exposure Disrupts Shh Secretion}

Alcohol exposure inhibits Shh in the Golgi from entering transport vesicles and therefore prevents the formation of $\mathrm{Shh} /$ caveolin-1 protein complexes in lipid raft domains, leading to defective transport of the ligand to the plasma membrane and resulting in Shh accumulation in the cytoplasm. We deduced that defective intracellular transport of Shh can lead to decreased secretion of the Shh ligand into the extracellular matrix. To confirm this hypothesis, we focused on the effect of alcohol exposure on Shh accumulation in the medium of a cultured HSC8B cells. We analyzed proteins collected from the HSC8B culture medium for Shh ligand content using two independent methods: western blot analysis and ELISA assay. When cell density in culture dishes reached $75 \%$ confluence, the cells were exposed to different concentrations of alcohol $(0,0.2,0.4,0.6$, and $0.8 \% \mathrm{w} / \mathrm{v}$ corresponding to $0,43,87,130$, and $174 \mathrm{mM}$ ), for $3 \mathrm{~h}$ in fresh DMEM medium containing $1 \%$ FBS. The cells were then incubated in alcohol-free DMEM medium containing $1 \%$ FBS for an additional $16 \mathrm{~h}$ to allow Shh secretion. The collected media were filtered through Millipore Centricon Plus100 to remove proteins having molecular weights higher than $100 \mathrm{kDa}$, and were then enriched, using the Centricon Plus- 
10 filtration system, for proteins having molecular weights ranging from 10 to $100 \mathrm{kDa}$. As shown in Figure 6a, western blot analysis of these proteins indicated that alcohol inhibits Shh secretion in a dose-dependent manner; ELISA assays indicated similar results. In detail, exposure of HSC8B cells to $0.2,0.4,0.6$, and $0.8 \%(43,87,130$, and $174 \mathrm{mM})$ alcohol concentrations corresponded to 1.1-, 1.7-, 2.9-, and 5.3-fold decreases in Shh secretion (Figure 6b). Western blot and ELISA experiments were performed in triplicate. To determine whether reduced Shh secretion might be due to cell death, cells were treated with different concentrations of alcohol $(0.2-1.6 \%)$ for 1 and $4 \mathrm{~h}$, and then assayed for caspase 3/7 apoptotic activity measurements. No significant differences were observed between the treated and untreated groups when alcohol concentrations were under $0.8 \%(\mathrm{w} / \mathrm{v})$, but at alcohol concentrations above $1.6 \%(\mathrm{w} / \mathrm{v}) 4$-h treat-
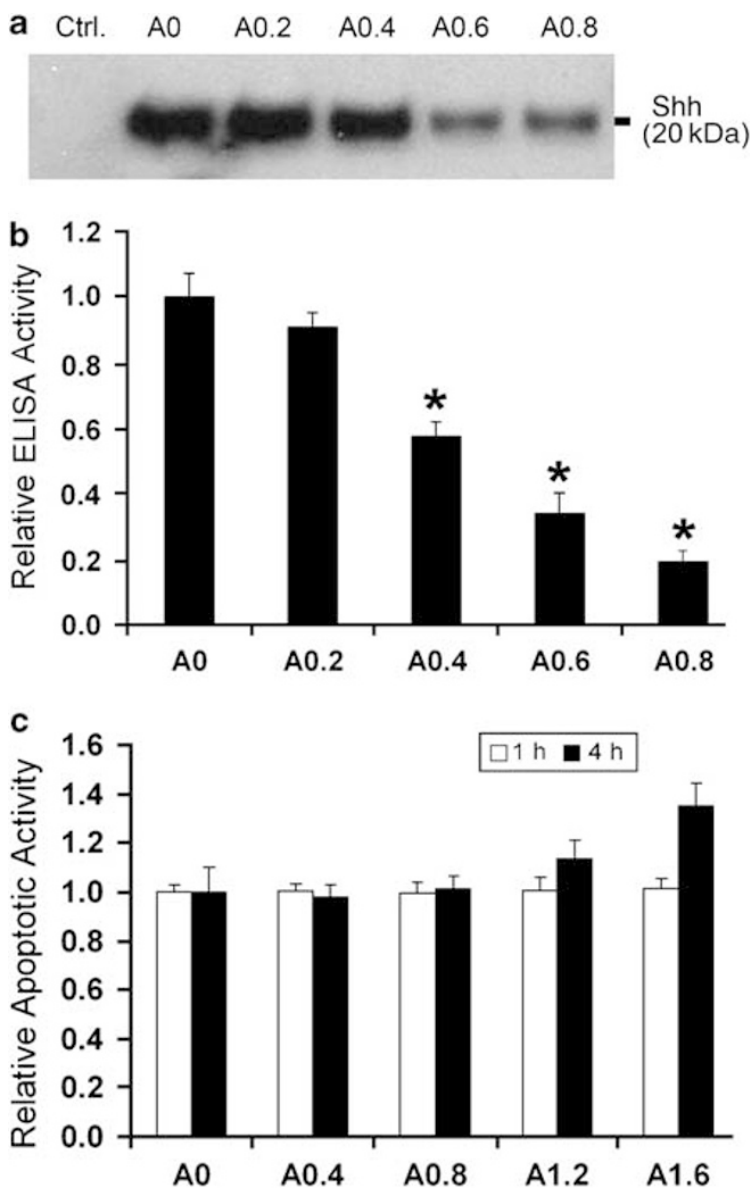

Figure 6 Alcohol exposure disrupts Shh secretion in a dose-dependent manner. Cultured HSC8B cells were exposed to various alcohol concentrations $(0,0.2,0.4,0.6$, and $0.8 \% \mathrm{w} / \mathrm{v})$ for $3 \mathrm{~h}$, after which media were collected and concentrated for analysis of Shh levels by western blot (a, medium alone serves as empty control) and ELISA assays (b). (c) Cell apoptotic activities have been measured by caspase 3/7 apoptosis assay under different concentrations of alcohol exposure for 1 and $4 \mathrm{~h}$. All of the experiments were repeated three times. Bars represent mean \pm s.e.m. ${ }^{\star} p<0.05$. ments caused a small increase in cell apoptotic activity (Figure 6c).

Our results indicate that alcohol inhibition of the hedgehog signal-transduction pathway is mediated through inhibition of the cholesterol modification of Shh, hindering Shh binding to caveolin-1 and preventing entry of Shh into transport vesicles from the trans-end of the Golgi apparatus. This results in an accumulation of Shh in the cytoplasm. Therefore, less Shh reaches the plasma membrane, leading to a deficiency of ligand secretion into the extracellular matrix.

\section{DISCUSSION}

Recently, we observed that physiological amounts of alcohol cause defects in cellular differentiation and organogenesis in a zebrafish model of FASD. The affected tissues and organs in FASD and in our zebrafish model are all targets of hedgehog signaling regulation during development. Transient loss of Hh signal transduction during specific developmental stages can result in permanent morphological abnormalities in these organs. Alcohol exposure results in defective posttranslational cholesterol modification of Shh, leading to a decreased association of mature Shh ligand with the plasma membrane. ${ }^{26}$ In this study, we further illuminate the effects of alcohol on the mechanisms that mediate Shh ligand intracellular transport and secretion. First, we have determined that caveolin-1 physically interacts with the Shh ligand to form a protein complex. Second, we have determined that alcohol exposure does not significantly disrupt Shh mRNA translated into Shh protein in the ER or the trafficking of Shh from the ER to the Golgi apparatus, and that alcohol exposure specifically causes a decrease in the amount of the caveolin-1/Shh complex found in lipid rafts. Third, alcohol treatment prevents the entry of Shh into vesicles that mediate transport from the Golgi to the plasma membrane, causing Shh to accumulate in the cytoplasm and leading to a deficiency of Shh secretion into the extracellular matrix (Figure 7).

During embryogenesis, foregut ventral endoderm cells that express high levels of Shh prior to mouse embryonic day $8.5^{31}$ will ultimately be involved in liver development; ventral endoderm cells that do not express Shh are specified to pancreatic lineages. ${ }^{32,33}$ During the developmental window starting at embryonic day 12 and lasting until day 16, hepatoblasts proliferate rapidly and differentiate into hepatocytes and cholangiocytes. Simultaneously, the expression levels of Shh and Ptc in these cells gradually decrease until they are undetectable on day 16. In the adult liver, Shh and other components of the Hh signaling pathway are found at levels that are barely detectable by immunostaining. However, using RT-PCR, expression of the genes that encode these proteins can be detected in purified stellate cells, immature cholangiocytes, and hepatic progenitor cells. Recently, we found that Shh and Hh signaling is involved in the molecular pathology of liver disease in animal model systems. Shh is an autocrine viability factor for myofibroblastic hepatic stellate 


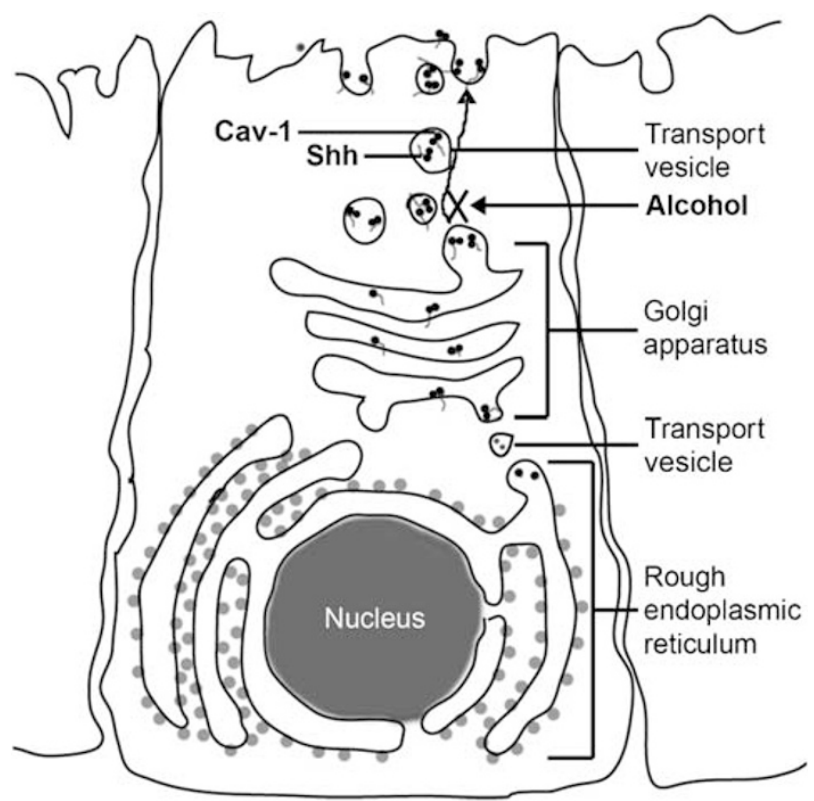

Figure 7 A model for Shh intercellular trafficking. Shh mRNA is translated in rough endoplasmic reticulum and transported into the Golgi apparatus. The maturation of Shh precursor takes place in the Golgi apparatus by proteolysis and lipid modifications. Cholesterol-modified Shh interacts with caveolin-1 and is packed as transport vesicles budding out from the transend of Golgi apparatus. These transport vesicles deliver the mature Shh ligand into the lipid raft domain on the plasma membrane for secretion. Alcohol exposure does not significantly inhibit Shh mRNA translation in rough endoplasmic reticulum and its trafficking from ER to Golgi, but specifically interrupts Shh entering the transport vesicle from the Golgi, causing Shh accumulation in the cytoplasm that leads to decreased secretion into the cellular matrix.

cells. ${ }^{27}$ Activation of hepatic stellate cells correlates with increased expression of Shh and Hh pathway components, and decreased expression of the Hh inhibitor, Hip. Increased Shh expression is induced by platelet-derived growth factor (PDGF) and is mediated through AKT. Factors, such as PDGF, that are released during liver injury promote the accumulation of myofibroblastic hepatic stellate cells, which can lead to cirrhosis of the liver. ${ }^{34}$ Hedgehog mediates mesenchymal-epithelial interactions that modulate hepatic responses to bile duct ligation. ${ }^{35} \mathrm{Hh}$ signaling also regulates epithelial-mesenchymal transitions during biliary fibrosis in rodents and humans. ${ }^{36}$ Following biliary duct injury, the activation of the $\mathrm{Hh}$ signaling pathway during cholestasis regulates ductular cell accumulation. ${ }^{37}$ Human hepatocarcinogenesis can involve defective regulation of the $\mathrm{Hh}$ signaling pathway. ${ }^{38}$ The maintenance of resident hepatic progenitors requires hedgehog signaling. ${ }^{39}$ The accumulation of hedgehog-responsive hepatic progenitor cells parallels the severity of alcoholic liver disease in mice and in humans.

The most common alcoholic liver diseases include fatty liver or steatosis, which can ultimately progress to cirrhosis. Most animals that chronically consume alcohol are more susceptible to steatosis than to fibrosis. Our finding of alcohol interrupts Shh transportation and secretion will help dissect out the Hh signaling defect in fetal alcohol syndrome. Furthermore, our study may provide insights into the pathological processes that lead to alcoholic liver diseases.

The Hh ligand is a morphogen that has a significant impact on embryonic development in species ranging from Drosophila melanogaster to Homo sapiens. Morphogens are factors that guide morphogenesis of a developmental field in a concentration-dependent manner. Cells in a single development field can differentiate into a variety of cell lineages depending on their positions in the field and the gradient concentration of the morphogen. ${ }^{40}$ Therefore, the intercellular transport and intracellular trafficking of Hh ligands have attracted a significant amount of attention from developmental biologists. For instance, it has been determined that release of the Hh ligand from the plasma membrane requires the Dispatched (Disp) protein. Disp is a transmembrane protein, having a sterol-sensitive domain that is required for release of the cholesterol-anchored Hh ligand. A glycosyl-phosphatidylinositol (GPI) moiety can replace cholesterol in effectively tethering Shh to the surface of expressing cells; however, Shh-GPI cannot be liberated from the cell membrane, indicating that cholesterol is an essential determinant of the Disp-dependent release mechanism of $\mathrm{Hh}$ ligand tethered to the cell membrane. ${ }^{41}$

Several proteins in tissues that respond to Hh signaling affect the range and distribution of $\mathrm{Hh}$ after it is released from secreting cells. These proteins include those in the heparan sulfate proteoglycan (HSPGs) protein family, which include those proteins encoded by dally and dally-like $(d l p) .{ }^{42,43}$ HSPGs contribute to Hh transport and are essential for establishing the $\mathrm{Hh}$ concentration gradient in a developmental field during embryogenesis. ${ }^{44,45}$ More importantly, the cholesterol modification of Hh affects the spread of $\mathrm{Hh}$ within tissues. Gallet et $a l^{46}$ found that cholesterol is required for long-range signaling; however, Callejo et $a l^{47}$ have reported that the absence of cholesterol modification results in widespread activation of two $\mathrm{Hh}$ targets. Determining the cause of these seemingly contradictory observations will require further study; however, it is clear that the cholesterol modification of Shh plays an important role in the intercellular transportation. We have now found that even prior to secretion into the extracellular matrix, cholesterol modification plays an important role in the intracellular transportation of Shh, particularly from the trans-end of the Golgi apparatus to the plasma membrane. We have identified that a key step in the intracellular transport of Shh is the formation of a protein complex with caveolin-1, which we have shown to be required for the destination of Shh located in the lipid raft of the plasma membrane. To our knowledge, we are the first to report that caveolin-1 is involved in the intracellular transport of the Shh.

The cells of caveolin-1-null mutant mice lack caveolae organelles. Surprisingly, these mice are viable and fertile. ${ }^{48,49}$ In striking contrast, zebrafish lacking caveolin-1 display 
significant developmental abnormalities and do not survive embryogenesis. ${ }^{50}$ Targeted depletion of Cav-1, using antisense morpholino oligomers, results in a substantial loss of caveolae and dramatic neural, eye, and somite defects in zebrafish. Interestingly, these phenotypes are very similar to the phenotypes caused by defective Shh signaling and developmental malformations induced by alcohol exposure. The variation in the severity of the phenotypes caused by careolin-1 deficiency in mammals and zebrafish can be explained by differences in the proteomes of fish and mammals. Mammalian genomes contain three genes that each encodes a different caveolin isoform: caveolin-1, caveolin-2, and caveolin-3. Most likely, null mutants for any one of these isoforms do not display severe phenotypes because the functions of the deleted isoform can be compensated for by the other isoforms. Zebrafish genomes express only a single caveolin isoform, homologous to mammalian Cav-1. Although alternate mechanisms for Shh intracellular transport may exist in species other than zebrafish, the existing evidence indicates that the key components of Shh transport are highly conserved during evolution. Disp, a protein involved in the release of the $\mathrm{Hh}$ ligand from the membrane, is highly evolutionarily conserved in species that range from $D$. melanogaster to $H$. sapiens. Shh sequences are highly conserved between species, particularly the amino-acid sequences (SGGCF) required for cholesterol modification of the protein, which are identical in zebrafish, mouse, and humans. Finally, cholesterol modification of the Shh ligand is necessary not only to activate the ligand but also for Shh transportation and gradient establishment.

It had been reported that the Hh receptor Ptc is associated with caveolin-1 in cholesterol-rich lipid raft microdomains of the plasma membrane, which may be related to the sequestration of Hh signaling. ${ }^{25}$ One way cells reduce the level of Shh signaling is by internalizing the Shh/Ptc complex through endocytosis, followed by proteolysis of the ligand. Here, we report that the Shh ligand also forms a protein complex with caveolin-1. This complex is required for Shh intracellular trafficking and for enriching cell membrane lipid raft domains with Shh. Our findings indicate that Hh ligand intracellular trafficking and secretion is probably one of the primary functions of caveolin-1. This observation is also in agreement with results from a previous report that showed that the sterol-linked hedgehog $\mathrm{N}$-terminal fragment associates specifically with membrane lipid rafts in Drosophila, suggesting that sterol modification is a novel mechanism for targeting proteins to raft membranes and that polarized intracellular transport of hedgehog requires lipid raft association. ${ }^{28}$ Indeed, this mechanism has been confirmed using a bio-engineered green fluorescent protein (GFP)/hedgehog C-terminal fragment fusion protein; this hedgehog C-terminal fragment leads to a hedgehog-like, autoprocessing reaction and produces a cholesterol modification of GFP, which results in high affinity of the modified GFP with plasma membranes. ${ }^{51}$
In summary, we have shown that under normal circumstances, the mature Shh ligand binds to caveolin-1, forming a protein complex in the Golgi apparatus, and enters the transport vesicles for delivery in lipid raft domains to the plasma membrane. Furthermore, alcohol exposure decreases the formation of Shh/caveolin-1 complexes in lipid rafts, consequently leading to the accumulation of Shh in the cytoplasm and deficient secretion into the extracellular matrix.

\section{ACKNOWLEDGEMENTS}

We thank Dr R Jhaveri, Dr DP Morris and Dr M Farrell for constructive criticisms and discussion during the preparation of this article. This study was supported by NIH AA016144 (Y-XL), NIH AA016001 (Y-XL), AHA 0430250N (Y-XL), and NIH AA010154 (AMD).

1. Jones KL, Smith DW. Recognition of the fetal alcohol syndrome in early infancy. Lancet 1973;2:999-1001.

2. Hammerschmidt M, Brook A, McMahon AP. The world according to hedgehog. Trends Genet 1997;13:14-21.

3. Nybakken K, Perrimon N. Heparan sulfate proteoglycan modulation of developmental signaling in Drosophila. Biochim Biophys Acta 2002;1573:280-291.

4. Stone DM, Hynes $M$, Armanini $M$, et al. The tumour-suppressor gene patched encodes a candidate receptor for Sonic hedgehog. Nature 1996;384:129-134.

5. Ding Q, Fukami S, Meng X, et al. Mouse suppressor of fused is a negative regulator of sonic hedgehog signaling and alters the subcellular distribution of Gli1. Curr Biol 1999:9:1119-1122.

6. Concordet JP, Lewis KE, Moore JW, et al. Spatial regulation of a zebrafish patched homologue reflects the roles of sonic hedgehog and protein kinase $A$ in neural tube and somite patterning. Development 1996;122:2835-2846.

7. Barth KA, Wilson SW. Expression of zebrafish nk2.2 is influenced by sonic hedgehog/vertebrate hedgehog-1 and demarcates a zone of neuronal differentiation in the embryonic forebrain. Development 1995;121:1755-1768.

8. Ericson J, Rashbass P, Schedl A, et al. Pax6 controls progenitor cell identity and neuronal fate in response to graded Shh signaling. Cell 1997;90:169-180.

9. Ingram WJ, Wicking CA, Grimmond SM, et al. Novel genes regulated by Sonic hedgehog in pluripotent mesenchymal cells. Oncogene 2002;21:8196-8205.

10. Porter JA, Young KE, Beachy PA. Cholesterol modification of hedgehog signaling proteins in animal development. Science 1996;274:255-259.

11. Mann RK, Beachy PA. Cholesterol modification of proteins. Biochim Biophys Acta 2000;1529:188-202.

12. Pepinsky $R B$, Zeng $C$, Wen $D$, et al. Identification of a palmitic acidmodified form of human Sonic hedgehog. J Biol Chem 1998;273:14037-14045.

13. Chen MH, Li YJ, Kawakami T, et al. Palmitoylation is required for the production of a soluble multimeric hedgehog protein complex and long-range signaling in vertebrates. Genes Dev 2004;18:641-659.

14. Zeng $X$, Goetz JA, Suber $L M$, et al. A freely diffusible form of Sonic hedgehog mediates long-range signaling. Nature 2001;411:716-720.

15. Feng J, White B, Tyurina OV, et al. Synergistic and antagonistic roles of the Sonic hedgehog $\mathrm{N}$ - and C-terminal lipids. Development 2004;131:4357-4370.

16. Cooper MK, Porter JA, Young KE, et al. Teratogen-mediated inhibition of target tissue response to Shh signaling. Science 1998;280:1603-1607.

17. Opitz JM, Penchaszadeh VB, Holt MC, et al. Smith-Lemli-Opitz (RSH) syndrome bibliography: 1964-1993. Am J Med Genet 1994;50: 339-343.

18. Opitz JM, de la Cruz F. Cholesterol metabolism in the RSH/SmithLemli-Opitz syndrome: summary of an NICHD conference. Am J Med Genet 1994;50:326-338.

19. Gaoua W, Wolf C, Chevy F, et al. Cholesterol deficit but not accumulation of aberrant sterols is the major cause of the teratogenic 
activity in the Smith-Lemli-Opitz syndrome animal model. J Lipid Res 2000;41:637-646.

20. Smart EJ, Ying YS, Donzell WC, et al. A role for caveolin in transport of cholesterol from endoplasmic reticulum to plasma membrane. J Biol Chem 1996;271:29427.

21. Fielding CJ, Bist A, Fielding PE. Intracellular cholesterol transport in synchronized human skin fibroblasts. Biochemistry 1999;38:2506.

22. Roy $S$, Luetterforst $R$, Harding $A$, et al. Dominant-negative caveolin inhibits $\mathrm{H}$-Ras function by disrupting cholesterol-rich plasma membrane domains. Nat Cell Biol 1999;1:98.

23. Galbiati F, Volonte D, Engelman JA, et al. Targeted downregulation of caveolin-1 is sufficient to drive cell transformation and hyperactivate the p42/44 MAP kinase cascade. EMBO J 1998;17:6633.

24. Scheel J, Srinivasan J, Honnert U, et al. Involvement of caveolin-1 in meiotic cell-cycle progression in Caenorhabditis elegans. Nat Cell Biol 1999;1:127-129.

25. Karpen HE, Bukowski JT, Hughes T, et al. The Sonic hedgehog receptor patched associates with caveolin-1 in cholesterol-rich microdomains of the plasma membrane. J Biol Chem 2001;276:19503-19511.

26. Li YX, Yang HT, Zdanowicz $M$, et al. Fetal alcohol exposure impairs hedgehog cholesterol modification and signaling. Lab Invest 2007;87:231-240.

27. Sicklick JK, Li YX, Choi SS, et al. Role for hedgehog signaling in hepatic stellate cell activation and viability. Lab Invest 2005;85:1368.

28. Rietveld A, Neutz S, Simons K, et al. Association of sterol- and glycosylphosphatidylinositol-linked proteins with Drosophila raft lipid microdomains. J Biol Chem 1999;274:12049.

29. Sicklick JK, Li YX, Choi SS, et al. Role for hedgehog signaling in hepatic stellate cell activation and viability. Lab Invest 2005;85:1368-1380.

30. Gallet A, Rodriguez R, Ruel $L$, et al. Cholesterol modification of hedgehog is required for trafficking and movement, revealing an asymmetric cellular response to hedgehog. Developmental Cell 2003:4:191-204.

31. Pepicelli CV, Lewis PM, McMahon AP. Sonic hedgehog regulates branching morphogenesis in the mammalian lung. Current Biology 1998;8:1083-1086.

32. Bort $R$, Signore $M$, Tremblay $K$, et al. Hex homeobox gene controls the transition of the endoderm to a pseudostratified, cell emergent epithelium for liver bud development. Dev Biol 2006;290:44.

33. Deutsch $G$, Jung J, Zheng $M$, et al. A bipotential precursor population for pancreas and liver within the embryonic endoderm. Development 2001;128:871-881.

34. Yang $L$, Wang $\mathrm{Y}, \mathrm{Mao} \mathrm{H}$, et al. Sonic hedgehog is an autocrine viability factor for myofibroblastic hepatic stellate cells. J Hepatol 2008;48:98-106.

35. Omenetti A, Yang L, Li YX, et al. Hedgehog-mediated mesenchymalepithelial interactions modulate hepatic response to bile duct ligation. Lab Invest 2007:87:499-514.
36. Jung YM, McCall SJ, Li YX, et al. Bile ductules and stromal cells express hedgehog ligands and/or hedgehog target genes in primary biliary cirrhosis. Hepatology 2007;45:1091-1096.

37. Omenetti A, Popov $Y$, Jung $Y$, et al. The hedgehog pathway regulates remodelling responses to biliary obstruction in rats. Gut 2008;57:1275-1282.

38. Sicklick JK, Li YX, Jayaraman A, et al. Dysregulation of the hedgehog pathway in human hepatocarcinogenesis. Carcinogenesis 2006; 27:748-757.

39. Sicklick JK, Li YX, Melhem A, et al. Hedgehog signaling maintains resident hepatic progenitors throughout life. Am J Physiol Gastrointest Liver Physiol 2006;290:G859-G870.

40. Guerrero I, Chiang C. A conserved mechanism of hedgehog gradient formation by lipid modifications. Trends Cell Biol 2007;17:1.

41. Burke R, Nellen D, Bellotto $M$, et al. Dispatched, a novel sterol-sensing domain protein dedicated to the release of cholesterol-modified hedgehog from signaling cells. Cell 1999;99:803-815.

42. Bornemann DJ, Duncan JE, Staatz W, et al. Abrogation of heparan sulface synthesis in Drosophila disrupts the Wingless, Hedgehog and Decapentaplegic signaling pathways. Development 2004;131: 1927-1938.

43. Takeo S, Akiyama T, Firkus $C$, et al. Expression of a secreted form of Dally, a Drosophila glypican, induces overgrowth phenotype by affecting action range of Hedgehog. Dev Biol 2005;284:204-218.

44. Han C, Belenkaya TY, Khodoun M, et al. Distinct and collaborative roles of Drosophila EXT family proteins in morphogen signalling and gradient formation. Development 2004;131:1563-1575.

45. Han CH, Belenkaya TY, Wang B, et al. Drosophila glypicans control the cell-to-cell movement of Hedgehog by a dynamin-independent process. Development 2004;131:601-611.

46. Gallet A, Ruel L, Staccini-Lavenant L, et al. Cholesterol modification is necessary for controlled planar long-range activity of Hedgehog in Drosophila epithelia. Development 2006;133:407-418.

47. Callejo A, Torroja C, Quijada L, et al. Hedgehog lipid modifications are required for Hedgehog stabilization in the extracellular matrix. Development 2006;133:471-483.

48. Drab $\mathrm{M}$, Verkade $\mathrm{P}$, Elger $\mathrm{M}$, et al. Loss of caveolae, vascular dysfunction, and pulmonary defects in caveolin-1 gene-disrupted mice. Science 2001;293:2449.

49. Razani B, Engelman JA, Wang XB, et al. Caveolin-1 null mice are viable but show evidence of hyperproliferative and vascular abnormalities. J Biol Chem 2001;276:38121-38138.

50. Fang PK, Solomon KR, Zhuang L, et al. Caveolin-1alpha and -1beta perform nonredundant roles in early vertebrate development. Am J Pathol 2006;169:2209-2222.

51. Vincent S, Thomas A, Brasher B, et al. Targeting of proteins to membranes through hedgehog auto-processing. Nat Biotechnol 2003:21:936-940. 